\title{
Pharmacokinetic considerations as to when to use dried blood spot sampling
}

In the past few years there has been a large increase in the reporting of the use of dried blood spots (DBS) in drug development. Most of these reports pertain to the technological improvements that have allowed for drug concentration measurements from microliter volumes of sample, issues concerning method development, and exploration of the technique, into other areas such as measurement of macromolecules and metabolite identification. One area that has received less attention and is the subject of this commentary concerns the pharmacokinetic issues that arise from using DBS as opposed to plasma, the mainstay matrix. Measurements of drug concentrations from either plasma or DBS are almost always the sum of bound and unbound drug, but it is the unbound drug in plasma (plasma water) that is the relevant driver of essentially all pharmacokinetic and pharmacodynamic events. Therefore, the critical assumption made is constancy in fraction unbound for plasma, and additionally for blood, constancy of hematocrit and blood cell affinity. Often these assumptions are reasonable and either matrix suffices, but not always. Then the value of one matrix over the other depends on the magnitude of the blood-to-plasma concentration ratio of drug, its clearance and the cause of the deviation from constancy. Additional considerations are the kinetics of distribution within blood and those arising when the objective is assessment or comparison of bioavailability. Most of these issues can be explored and addressed in vitro prior to the main drug development program.

Keywords: blood $\approx$ blood cell distribution $=$ concentration $\approx$ dried blood spot $\approx$ hematocrit " pharmacodynamic $=$ pharmacokinetic $\|$ unbound drug

The use of dried blood spots (DBS) for drug concentration sampling has received much interest in the past year [1-5]; as attested to by the devotion of a second full issue of this journal to the topic. The approach had its origin in the work of Guthrie with development of a simple method for measuring inborn errors of metabolism in neonates [6]. Subsequent to the development of the so-named Guthrie test, DBS has been used for collection of blood samples for the measurement of specific antibodies $[7,8]$, viruses $[9,10]$ and proteins [11,12] by using ELISA, PCR and protein activity assays.

\section{“"Think ahead when considering the strategy."}

In recent years, improvements in bioanalytical detection have allowed for measurement of drug from DBS obtained from only $4-12 \mu$ of blood [13]. In addition, improvements in paper quality for the untreated stock has expanded the usefulness of the technique, providing additional options for quantitative analysis, in addition to the treated paper already available for method development [14]. Many analytical obstacles have already been addressed confirming DBS as a viable approach for quantitative measurement of drugs and metabolites [4,15-17]. DBS has been shown to be useful at various stages of research/development, with applicability in discovery [18], preclinical development [19] and human sampling [20]. Continued improvements in the technologies associated with the technique, such as the use of ultra-performance LC [21], dispersion spray MS [22], direct desorption [23], online desorption $[24,25]$ and card handling and extraction automation, are expected to increase the use of this approach.

Distinct practical advantages have been demonstrated for the use of DBS in place of plasma in drug development. These include reductions in: the sample volumes required, the number of animals required for pharmacokinetic (PK) studies, and the cost of shipping/

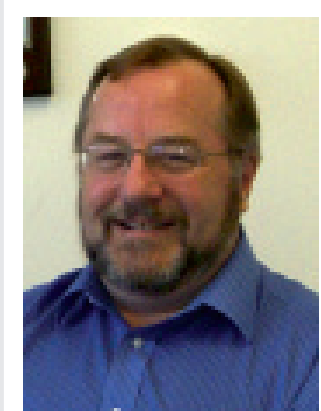

Gary Emmons

Author for correspondence:

Drug Disposition, Safety \& Animal

Research, sanofi-aventis,

Bridgewater, NJ, USA

E-mail: gary.emmons@

sanofi-aventis.com

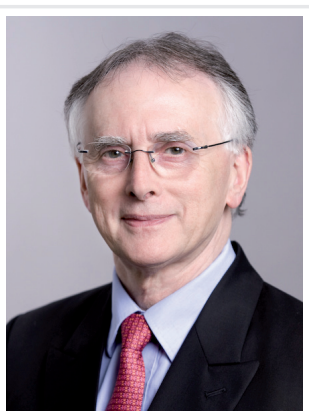

Malcom Rowland

School of Pharmacy \&

Pharmaceutical Sciences, University of Manchester, Manchester, UK 
storage of samples from the site of collection to the analytical laboratory. Storage of samples collected on filter paper can be managed at room temperature and shipment does not require, as is common for plasma, dry ice. In a recent publication, the cost savings by not shipping PK samples on dry ice in a large clinical trial were estimated to be in the tens of thousands of euros [1].

"Measurements of drug concentrations
from either plasma or dried blood spots are
almost always the sum of bound and unbound
fractions, but it is the unbound fraction in
plasma (plasma water) that is the relevant
driver of essentially all pharmacokinetic
and pharmacodynamic events."

Although much has been written about overcoming the analytical obstacles and practical advantages to using the DBS technique, there has not been much attention given to the PK and pharmacodynamic (PD) issues raised by using this approach. Plasma has been the matrix of choice for many years and the associated issues raised by the use of DBS were explored in a recent publication [26] and are continued here.

One aspect of DBS sampling that should not be overlooked is that the low volume requirements for blood sampling provide an opportunity for obtaining samples by heel or finger prick. In fact, it is this possibility that is exploited in examples of DBS-based therapeutic drug monitoring, some of which include sampling by the patient at home. Since the initial flow from such a 'prick' is contaminated with interstitial fluid, good practice warrants the discarding of the first drop. Capillary blood, as obtained by heel or finger pricking, is a mixture of venous and arterial blood and generally, especially if the site has been immersed in warm water to facilitate blood flow, more reflective of arterial blood. Given the arterial component and the fact that collection is usually at a site different from that used in normal venous sample collection, there may be differences in drug concentration between the two [27]. However, such differences between venous and capillary blood drug concentrations are likely to only be seen shortly after drug administration. A practical matter concerning bioanalytical method development is that these samples, if applied directly to the filter paper, will not contain anticoagulant while the standards and quality control samples used for quantitation of the incurred sample will. Another practical matter if the samples are taken directly from animals is the potential for contamination from dietary or excretory sources.

\section{"Distinct practical advantages have been demonstrated for the use of DBS in place of plasma in drug development."}

Dried blood spot samples are in essence the same as whole blood samples and have most of the same issues as those of the matrix concerning estimation and interpretation of PK parameters. Total drug measurement (sum of bound and unbound drug) from either plasma or DBS should be considered a surrogate for the unbound plasma concentration $(\mathrm{Cu})$ of the drug, which is the more relevant driver than total concentration of essentially all events (PK and PD) within the body. Arguably, in certain circumstances measurement of drug in blood provides a more physiological basis than plasma for interpreting clearance [28]. However, blood has been a difficult matrix to work with. In addition, measurement of $\mathrm{Cu}$ has also proven difficult on a routine basis. For this reason plasma has been the mainstay matrix for measurement in drug discovery and development.

The key questions are therefore: when does either blood (i.e., DBS) or plasma cease to be an appropriate surrogate for $\mathrm{Cu}$ and, on such occasions, what steps are needed to make the appropriate correction? To address these questions we need to understand the relationship between $\mathrm{Cu}$ and total drug concentration in plasma and blood, displayed schematically in Figure I. At equilibrium the relationships for plasma (Equation I) and DBS (Equation 2) are:

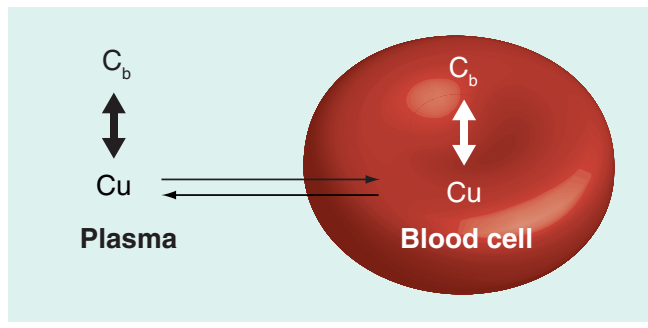

Figure 1. Events occurring within blood. The extent and kinetics of distribution into cells depends upon the permeability of the cell membrane and the affinity of compound for constituents within plasma and the blood cell.

$C_{b}$ : Bound concentration;

$\mathrm{Cu}$ : Unbound concentration. 


$$
\begin{gathered}
C=\frac{C u}{f u} \\
C b=\left[\frac{1-H}{f u}+H \cdot \rho\right] C u
\end{gathered}
$$

Equation I

EQUATION 2

where $\mathrm{Cb}$ is the blood concentration.

Thus, it is seen that total plasma concentration is directly proportional to $\mathrm{Cu}$ when the unbound fraction in plasma (fu) is constant. Likewise, total $\mathrm{Cb}$ is proportional to $\mathrm{Cu}$ when $\mathrm{fu}$, hematocrit $(\mathrm{H})$ and blood cell partitioning $(\rho)$ are constant. In many situations these conditions hold reasonably well. Then, either drug in plasma or DBS can be used to reflect the unbound drug concentration. However, there are some notable exceptions, which are now explored.

\section{"Dried blood spot samples are in essence the same as whole blood samples and have most of the same issues as those of the matrix concerning estimation and interpretation of pharmacokinetic parameters."}

The blood-to-plasma concentration ratio $(\mathrm{R})$ is a useful metric to evaluate the situation. Many drugs, especially hydrophilic ones, therapeutic proteins and acidic drugs with high affinity for plasma proteins, do not partition into erythrocytes, or do so minimally, and for such drugs $\mathrm{R}$ tends towards the lower limit of 0.55 to 0.60 . In these situations, blood cells simply act as a diluent, and the only concern over the usual one of altered plasma binding is the hematocrit, if DBS is used. Generally, the hematocrit is fairly constant at 0.45 in adult males, is often slightly lower in adult females, and is higher in newborns [29]. Concern for varying hematocrit can arise, however, when extrapolating PK interpretations from healthy individuals to patients with varying types or degrees of anemia, or in situations where the drug itself (or other concomitantly administered drugs) alters the hematocrit $[30,31]$.

Some drugs, such as caffeine and alcohol, enter erythrocytes but do not bind to proteins there or in plasma, and for these drugs the value of $\mathrm{R}$ is essentially one, and the total blood concentration is virtually the same as $\mathrm{Cu}$ (the small difference is due to the volume occupied by solid constituents of cells), and no concern for use of DBS exists.

For drugs that enter blood cells and bind both there and in plasma, it is useful to know which parameter, fu or $\rho$, is the more significant influencing the relationship between $\mathrm{Cu}$ and total concentration. When $\mathrm{R} \geq 1.5$, it is seen by dividing Equation 2 by Equation I that $\rho$ is greater than $1 /$ fu. Furthermore, under these circumstances, examination of EQuation 2 shows that blood concentration is relatively insensitive to changes in plasma protein binding, and that now $\rho$ becomes the most important parameter relating total blood to unbound plasma concentration. It is then critically important to understand the extent and sources of variability in $\rho$ if DBS is to be used. When there is minimal variability in $\rho$, then DBS is arguably preferred over plasma. However, for drugs such as cyclosporine and tacrolimus, in which $\mathrm{R}$ is more than two due to high affinity for intracellular erythrocyte binding sites, $\rho$ can and does change, for example, due to saturation of erythrocyte binding over the range of therapeutic concentrations, at least for cyclosporine [32]. In these situations, caution should be exercised in interpreting blood PK, although there still may be reasons for preferring blood over plasma. One reason is the hemolysis that can occur during preparation of plasma, which may introduce significant error into the measurement of plasma concentration due to interference or catalytic activity [33]. Evaluation of the presence of hemolysis in general practice is a subjective assessment. Nonetheless, assuming a tolerance of $10 \%$ is the allowed limit in the increase in plasma concentration due to hemolysis, calculation shows that the affinity of tacrolimus for erythrocytes is so high that this tolerance is met when only $0.5 \%$ hemolysis occurs.

\section{"One aspect of dried blood spot sampling \\ that should not be overlooked is that the low volume requirements for blood sampling provide an opportunity for obtaining samples by heel or finger prick."}

Another technical reason for the preference of blood over plasma for cyclosporine and tacrolimus is that erythrocyte binding of such compounds is temperature dependent [34], so that if one wished to obtain an estimate of the plasma concentration that existed in vivo at the time of collection, the blood sample would need to be kept at $37^{\circ} \mathrm{C}$ during the separation of plasma, which creates problems in routine clinical practice. Given these complexities for both drugs, blood, rather than plasma, has been used during drug development and in their subsequent therapeutic drug monitoring $[20,35]$. 
The distribution of the drug into erythrocytes is time dependent. Usually, this process is extremely fast and is of no practical concern. Occasionally, it is relatively slow, which may impact the interpretation of the data obtained by DBS or plasma. For example, it takes several minutes for distribution equilibrium to be achieved in the case of cyclosporine and related compounds [36], while it takes even longer for the carbonic anhydrase inhibitor chlorthalidone, with the blood cell concentration peaking several hours after plasma following drug administration, at which time most of the drug in blood is in the erythrocytes, avidly bound to the very high concentration of carbonic anhydrase there [37]. In such extreme situations, if the events of interest are driven by, or correlate with, unbound drug in plasma, before distribution equilibration is achieved, plasma may be a better matrix than DBS. Whereas, in the case of chlorthalidone, the erythrocyte is the site of action, and therefore DBS would be the more appropriate matrix. Collectively, these examples indicate that when $\rho$ is not constant one needs to consider and where necessary evaluate, each of the various factors that may lead to differences in $\rho$, such as time, temperature and concentration in addition to potential demographic covariates such as disease, age, genetics, gender and co-medications, if DBS is being considered.

In all situations, another consideration in the choice of the matrix when $\rho$ is not constant is the objective of the study. Estimating bioavailability is a key objective of many PK-related studies and the results can be influenced by the choice of plasma versus DBS. A common basis in the assessment of bioavailability is comparison of the AUC values between either an extravascular and intravenous dose, or two extravascular doses, with the key assumption that clearance is constant between treatments. If however this is not the case due, for example, to saturable binding/ partitioning into the erythrocytes, then clearance may change, and the ratio of DBS AUCs may no longer provide an accurate estimate of bioavailability. For example, with cyclosporine, $\rho$ demonstrates concentration dependency and plasma is the better choice of matrix for bioavailability determinations, as plasma clearance, but not blood clearance, is constant [38]. When, however, bioequivalence is the goal and the concentration-time profiles of the two formulations are similar, then either matrix is acceptable [35].

\section{Conclusion}

TABLE I summarizes the preceding commentary concerning the choice of matrix for drug concentration measurements. When variability in fu and $\rho$ is low, then either plasma or DBS can be used. When the blood-to-plasma ratio is close to 0.55 , then variability in fu and (to a lesser extent hematocrit) become the concern, and an understanding of the inter- and intra-individual variation in plasma protein binding is required. However, when the bloodto-plasma ratio is much larger, especially when two or greater, then variability in $\rho$ becomes the dominant concern when using DBS. If it can be demonstrated that there is little variability in $\rho$, then because of hemolysis concerns, the DBS approach is preferred over plasma, whilst always bearing in mind the objective of

\section{Table 1. Decision tree for choosing plasma or dried blood spot matrix.}

\begin{tabular}{|c|c|c|c|c|}
\hline Blood:plasma ratio & Hematocrit constant $^{\dagger}$ & fu constant & $\rho$ constant & Plasma and/or DBS \\
\hline $0.55<2.0$ & Y & Y & Y & Plasma or DBS can equally be used \\
\hline $0.55<2.0$ & Y & $\mathrm{N}$ & Y & $\begin{array}{l}\text { Plasma or DBS can be used, but the reasons for } \\
\text { variability in plasma protein binding need to be } \\
\text { understood and accommodated }\end{array}$ \\
\hline$\geq 2.0$ & Y & Y & Y & DBS is preferred due to hemolysis concerns \\
\hline$\geq 2.0$ & Y & $\mathrm{N}$ & Y & DBS is preferred due to hemolysis concerns \\
\hline Any & $\mathrm{N}$ & $\mathrm{Y} / \mathrm{N}$ & $\mathrm{Y} / \mathrm{N}$ & $\begin{array}{l}\text { Decision depends on value of } R \text { as in above scenarios with } \\
\text { the additional need to correct for hematocrit when using } \\
\text { DBS if } R \text { approaches } 0.55 \text { or } \geq 2.0\end{array}$ \\
\hline
\end{tabular}

${ }^{+}$By constant it is meant that the parameter value varies to a sufficiently small extent not to materially affect the relationship between unbound plasma and total concentrations.

DBS: Dried blood spot; fu: Unbound fraction in plasma; N: No; R: Blood-to-plasma concentration ratio; Y: Yes. 
the study. When variability in $\rho$ is large then caution should be exercised before using DBS. The magnitude and factors causing the variability in $\rho$, including time, temperature, and concentration effects, can often be evaluated in vitro and should be undertaken before choosing DBS during development of potential drug candidates. Unfortunately, to date there has been very little study of inter-and intra-subject variability in $\rho$.

\section{Financial \& competing interests disclosure}

The authors have no relevant affliations or financial involvement with any organization or entity with a financial interest in or financial conflict with the subject matter or materials discussed in the manuscript. This includes employment, consultancies, honoraria, stock ownership or options, expert testimony, grants or patents received or pending, or royalties. No writing assistance was utilized in the production of this manuscript.

\section{Bibliography}

1 Evans C. Dried blood spot analysis: a paradigm shift within the pharmaceutical industry. AAPS Newsmagazine, April 2010.

2 ter Heine R, Rosing H, van Gorp ECM, Mulder JW, Beijnen JH, Huitema ADR. Quantification of etravirine (TMC125) in plasma, dried blood spots and peripheral blood mononuclear cell lysate by liquid chromatography tandem mass spectrometry. J. Pharm. Biomed. Anal. 49, 393-400 (2009).

3 Liang X, Li Y, Barfield M, Ji QC. Study of dried blood spots techniques for the determination of dextromethorphan and its metabolite dextrorphan in human whole blood by LC-MS/MS. J Chromatog. B 877, 799-806 (2009).

4 Li W, Tse FLS. Dried blood spot sampling in combination with LC-MS/MS for quantitative analysis of small molecules. Biomed. Chromatog. 24, 49-65 (2010).

5 van der Heijden J, de Beer Y, Hoogtanders K et al. Therapeutic drug monitoring of everolimus using the dried blood spot method in combination with liquid chromatography-mass spectrometry. J. Pharm. Biomed. Anal. 50, 664-670 (2009).

6 Guthrue R, Suzi A. A simple phenylalanine method for detecting phenylketouria in large populations of newborn infants. Pediatrics 23, 338-343 (1963).

7 Tran, Thanh Nga T et al. Enzyme-linked immunoassay for dengue virus IgM and IgG antibodies in serum and filter paper blood. Biomed. Central Infectious Diseases 6, 13-20 (2006).

8 Hofmana L, Foleya TP, Henrya JJ, Naylora EW. The use of filter paper-dried blood spots for thyroid-antibody screening in adults. J. Lab. Clin. Med. 144, 307-312 (2004).
9 Lewensohn-Fuchs I, Osterwall P, Forsgren M, Malm G. Detection of herpes simplex virus DNA in dried blood spots making a retrospective diagnosis possible. J. Clin. Virol. 26, 39-48 (2003).

10 Stevens W, Erasmus L, Moloi M, Taleng T, Sarang S. Performance of a novel human immunodeficiency virus (HIV) type 1 total nucleic acid-based real-time PCR Assay using whole blood and dried blood spots for diagnosis of HIV in infants. J. Clin. Micr. 46, 3941-3945 (2008).

11 McDade TW, Burhop J, Dohnal J. Highsensitivity enzyme immunoassay for C-reactive protein in dried blood spots. Clin. Chem. 50, 652-654 (2004).

12 Bowlin FG, Brown ARD. Development of a protocol for newborn screening for disorders of the galactose metabolic pathway. J. Inher. Metab. Dis. 9, 99-104 (1986).

13 Spooner N, Lad R, Barfield M. Dried blood spots as a sample collection technique for the determination of pharmacokinetics in clinical studies: considerations for the validation of a quantitative bioanalytical method. Anal. Chem. 81, 1557-1563 (2009).

14 Mei JV, Alexander JR, Adam BW, Hannon WH. Use of filter paper for the collection and analysis of human whole blood specimens. J. Nutr. 131, 1631S-1636S (2001).

15 Edelbroeck PM, van der Heijden J, Stolk LML. Dried blood spot methods in therapeutic drug monitoring: methods, assays and pitfalls. Ther. Drug Monit. 31, 327-336 (2009).

16 Damen CWN, Rosing H, Schellens JHM, Biejnen JH. Application of dried blood spots combined with high-performance liquid chromatography coupled with electrospray ionization tandem mass spectrometry for simultaneous quantification of vincristine and actinomycin-D. Anal. Bioanal. Chem. 394, 1171-1182 (2009).
17 McDade TW, Williams S, Snodgrass JJ What a drop can do: dried blood spots as a minimally invasive method for integrating biomarkers into population-based research. Demography 44, 899-925 (2007).

18 Beaudette P, Bateman KP. Discovery stage pharmacokinetics using dried blood spots. J. Chromatog. B 809, 153-158 (2004).

19 Barfield M, Spooner N, Lad R, Parry S, Fowles S. Application of dried blood spots combined with HPLC-MS/MS for the quantification of acetaminophen in toxicokinetic studies. J. Chromatogr. B 870, 32-37 (2008).

20 Hoogtanders $\mathrm{K}$, van der Heijden J, Christiaans M, Edelbroeck P, van Hoof JP, Stolk LML. Therapeutic drug monitoring of tacrolimus with the dried blood spot method. J. Pharm. Biomed. Anal. 44, 658-664 (2007).

21 Al-Dirbashi OY, Rashed MS, Jacob M et al. Improved method to determine succinylacetone in dried blood spots for diagnosis of tyrosinemia type 1 using UPLC-MS/MS. Biomed. Chromatogr. 22, 1181-1185 (2008).

22 Wang H, Liu J, Cooks RG, Ouyang Z. Paper spray for direct analysis of complex mixtures using mass spectrometry. Angew. Chem. Int. Ed. 49, 877 -880 (2010).

23 Wiseman J, Evans CA, Bowen CL, Kennedy JH. Direct analysis of dried blood spots utilizing desorption electrospray ionization (DESI) mass spectrometry. Analyst 135, 720-725 (2010).

24 Déglon J, Thomas A, Cataldo A, Mangin P, Staub C. On-line desorption of dried blood spot: a novel approach for the direct LC/MS analysis of $\mu$-whole blood samples. J. Pharm. Biomed. Anal. 49, 1034-1039 (2009).

25 Abu-Rabie P, Spooner N. Direct Quantitative bioanalysis of drugs in dried blood spot samples using a thin-layer chromatography mass. spectrometer interface. Anal. Chem. 81, 10275-10284 (2009). 
26 Rowland M, Emmons G. Use of dried blood spots in drug development: pharmacokinetic considerations. AAPS J. 12(3), 290-293 (2010).

27 Chiou WL. the phenomenon and rationale of marked dependence of drug concentration on blood sampling site: implications in pharmacokinetics, pharmacodynamix, toxicology and therapeutics (Part I). Clin. Pharmacokinet. 17(3), 175-199 (1989).

28 Rowland M, Tozer TN. Clinical Pharmacokinetics: Concepts and Applications (4th Edition). Lippincott, Williams \& Wilkins, Baltimore, USA 157-168 (2010).

29 Lichtman M, Beutler E, Kaushansky K, Kipps T, Seligsohn U, Prchal J. Williams Hematology (7th Edition). McGraw-Hill Medical 12, 85 (2006).

30 Wood PA, Hrushesky JM. Cisplatinassociated anemia: an erythropoietin deficiency syndrome. J. Clin. Investig. 95, 1650-1659 (1995).
31 Innis JD, Hurwitz A. Mechanism of hematocrit increase induced by the combined administration of morphine and adriamycin: role of histamine release. Toxicol. Appl. Pharmacol. 90, 454-464 (1987).

32 Kawai R, Matthew D, Tanaka C, Rowland M. Physiologically-based pharmacokinetics of cyclosporine A: extension to tissue distribution kinetics in rats and scale-up to human. J. Pharmacol Expt. Therap. 287, 457-468 (1998).

33 Laessig RH, Hassemer DJ, Paskey T A, Schwartz TH. The effects of 0.1 and 1.0 per cent erythrocytes and hemolysis on serum chemistry values. Am. J. Clin. Pathol. 66, 639-644 (1976)

34 Tozer TN. Implications of altered plasma protein binding in disease states. In: Pharmacokinetic Basis of Drug Treatment. Benet LZ, Massoud N, Gambertoglio JG (Eds). Ravens Press, NY, USA, 173-193 (1983).
35 Mee AV, Wong PY, Sun C et al. Monitoring of cyclosporine concentrations by using dry blood-spot samples. J. Clin. Lab. Anal. 5, 74-77 (1991).

36 Kawai R, Lemaire M, Steimer J-L, Bruelisauer A, Niederberger W, Rowland M. Physiologically-based pharmacokinetic study on a cyclosporin derivative, SDZ IMM 125.

J. Pharmacokin. Pharmacodyn. 22, 327-365 (1994).

37 Fleuren HLJ, van Rossum M. Nonlinear relationship between plasma and red blood cell pharmacokinetics of chlorthalidone in man. J. Pharmacokin. Pharmacodyn. 5 , 359-375 (1977).

38 Legg B, Rowland M. Saturable binding of cyclosporin A to erythrocytes: estimation of binding parameters in renal transplant patients and implications for bioavailability assessment. Pharm. Res. 5, 80-85 (1988). 